\title{
Universal dynamo paradigm for solar activity, Higgs fields and disasters
}

\author{
Boris Shevtsov ${ }^{1, *}$ \\ ${ }^{1}$ Institute of Cosmophysical Research and Radio Wave Propagation, FEB RAS, 7, Mirnaya st., \\ Paratunka, Kamchatka, Russia, 684034
}

\begin{abstract}
There is still a problem of a correct and accurate description of the dynamo and its uses in various fields of physics. To solve this problem, a special and universal representation of dynamo is proposed. The magnetic induction equation of dynamo is presented in the form of a Lienard relaxation oscillator with cubic nonlinear restoring force corresponding to the Mexican hat or champagne bottle potential which is used to determine the Higgs fields which are considered here in its general sense. Universal dynamo paradigm in field theory which can be used to describe disasters is proposed. Using solar activity as an example, it is shown how a dynamo induces a magnetic analogue of the Higgs fields with a broken symmetry of the magnetic field. Various dynamo modes are considered and different dynamo numbers are estimated. The dynamo effect can be used in field theory as an alternative to spontaneous symmetry breaking. Opportunities for the promotion of the new dynamo paradigm are discussed.
\end{abstract}

\section{Introduction}

Many physical systems are represented by relaxation generators. An example of this is Dynamo, the development of the theory of which largely determines the state of modern physics. As another confirmation of the universality of relaxation mechanisms, let us consider the analogies between solar activity, Higgs fields and catastrophes that follow from the representation of a magnetic dynamo in the form of a multidimensional Lienard relaxation oscillator [1] with a cubic nonlinearity of the returning force corresponding to the Mexican hat or champagne bottle potential which is used to determine the Higgs fields and to discribe the disasters.

Along with the field generation, the dynamo effect gives a forced symmetry breaking, which can be considered as an alternative to the spontaneous breaking used in field theory. How this works at different scales will be shown by the example of solar activity, for which previously unknown features of nonlinear oscillations of the Lienard generator, Higgs fields and catastrophes will be obtained. It will be shown that the nonlinearity of oscillations increases at small scales, which justifies the use of the dynamo effect to explain the forced symmetry breaking in field theory. It is noteworthy that the nonlinearities of the dynamo are determined by the gravitational field, according to which it is possible to compare the mass of the object responsible for the dynamo effect.

\footnotetext{
*e-mail: bshev@ikir.ru
} 
The considered analogies give a dynamo paradigm of elementary particles, according to which particles are formed as fluctuations on different scales as a result of the dynamo effect. Elementary particles can be defined as the inner edge of the scaling, and the universe and galaxies as its outer edge. The dynamo effect consolidates naturally the physical movements on all scales that corresponds to the Mach principle. While independent motions on different scales correspond to the usual understanding of particles in the first approximation.

The disasters theory in the light of the dynamo paradigm can become an alternative to the Big Bang theory and a fruitful concept for understanding the sudden, rapid and spontaneous emergence of life at the molecular and cellular levels. The theory of catastrophes describes explosive processes and combines well the evolutionary and revolutionary phases of development. Inflation and collapse on the scales depend on the ratio of different types of energy, and the main blow of disasters is the embodiment of the singularity. If we know what galaxies and cyclones look like, then we can imagine what atoms and elementary particles look like in the theory of catastrophes with the dynamo paradigm according to the similarity principle.

The analogue of the Higgs boson can be represented as displaced weak oscillations, known in dynamo as excursions of magnetic poles. In this view, the displacement is explained by the dynamo effect, and not by a spontaneous breaking of symmetry. Highly nonlinear reversible oscillations can be compared to the generalized Higgs boson.

The development of the dynamo concept largely reflects the success of modern physics. A special representation of the dynamo by a Lienard relaxation oscillator with a cubic nonlinearity of the returning force opens up new opportunities in the development of field theory and allows us to revise numerous dynamo models in order to determine the effectiveness of various approximations.

The relaxation oscillator model was proposed in [2-4], and its development is in [5, 6]. A generalization to the multidimensional case, which can be used in nonlinear field theory, is considered in [1].

The idea of a relaxation generator in one form or another was used to create the theory of a magnetic dynamo [7-22] and see additionally the reviews [23-25], but there is no convection in the early models. The low-mode models with convection have a particular place in this theory [26-29], but only the representation of the dynamo in the form of the multidimensional Lienard oscillator [1] defines correctly the symmetry of the system and opens up new ways in the development of nonlinear physics thanks to the universality and productivity of the new dynamo paradigm and the specificity of relaxation oscillations.

The dynamo representation which will be discussed below can be called canonical because it is obtained without any approximations, reveals the mechanism of dynamo and takes into account all the nuances of field generation. It is not difficult to generalize the dynamo effect to any fields.

\section{Magnetic dynamo}

We write the equation of magnetic induction $\mathbf{B}$ in the form of an oscillator equation [? ]:

$$
\begin{gathered}
\partial_{t} \mathbf{B}=\mathbf{Y}, \\
\partial_{t} \mathbf{Y}=\operatorname{rot}\left(\partial_{t} \mathbf{v} \times \mathbf{B}+\mathbf{v} \times \mathbf{Y}\right)+v_{m} \Delta \mathbf{Y},
\end{gathered}
$$

where $\mathbf{v}$ is the velocity of a medium, $v_{m}=1 / \mu \mu_{0} \sigma$ is the magnetic viscosity, $\mu$ and $\mu_{0}$ are the relative and absolute magnetic permeabilities, $\sigma$ is the electrical conductivity of a medium, $\mathbf{Y}=\operatorname{rot}(\mathbf{v} \times \mathbf{B})+v_{m} \Delta \mathbf{B}$. 
We write the Navier-Stokes equation in the Boussinesq approximation as the diffusion equation:

$$
\begin{gathered}
\partial_{t} \mathbf{v}=\hat{D} \mathbf{v}+\mathbf{f} \\
\hat{D} \mathbf{v}=-\rho^{-1} \nabla P-2 \Omega \mathbf{e}_{z} \times \mathbf{v}-(\mathbf{v}, \nabla \mathbf{v})+v \Delta \mathbf{v}, \\
\mathbf{f}=g \beta T \mathbf{e}_{r}-\left(\rho \mu \mu_{0}\right)^{-1}(\mathbf{B} \times \operatorname{rot} \mathbf{B}),
\end{gathered}
$$

where $P$ is the pressure, $v$ is the kinematic viscosity, $\Omega$ is the angular velocity of the convective layer rotation, $\rho$ is the density of a medium, $g$ is the acceleration of gravity, $\beta$ is the coefficient of thermal volumetric expansion, $\mathbf{e}_{z}$ and $\mathbf{e}_{r}$ are the unit vectors of a spherical coordinate system, $T$ is the temperature deviation from the stationary value $T_{0}$ in the convection layer at $\mathbf{v}=0$.

The solution of equations (2) in the form of the Stokes' law

$$
\mathbf{v}(\mathbf{r}, t)=\hat{G} \mathbf{f}(\mathbf{r}, t)
$$

allows us to rewrite first equation (2) as

$$
\partial_{t} \mathbf{v}=(\hat{D} \hat{G}+\hat{1}) \mathbf{f},
$$

where $\hat{1}$ is the unit operator.

Substituting (3) and (4) in (1), we obtain the equation of magnetic induction in the form of the multidimensional Lienard equation $[1,5]$ :

$$
\begin{gathered}
\partial_{t} \mathbf{B}=\mathbf{Y} \\
\partial_{t} \mathbf{Y}=\operatorname{rot}[\hat{G} \mathbf{f} \times \mathbf{Y}+(\hat{D} \hat{G}+\hat{1}) \mathbf{f} \times \mathbf{B}]+v_{m} \Delta \mathbf{Y}
\end{gathered}
$$

Equations (5) are a symbolic notation of the magnetic induction equation with integral operator kinematic coefficients $\hat{D}$ and $\hat{G}$. It generalises the concept of dynamo numbers, the physical meaning of which is revealed depending on problem conditions.

The $\hat{D} \hat{G}$ composition in (4) and (5) defines kinematic waves correlations and specifics of their oscillations. $\hat{D}$ represents diffusion and scales consolidation. $\hat{G}$ represents turbulence and scales fragmentation. $\hat{D} \hat{G}$ combines two processes.

The magnetic feedback $\mathbf{f}$, which is a balans between Archimedes and Lorentz forces, affects damping and frequencies of oscillations. The attenuation and amplification of the magnetic field is determined by $\hat{G} g \beta T \mathbf{e}_{r}$. This kinematic temperature tandem is a convection wave.

The equations (5) are a multidimensional genearalisation of Van der Pol [2-4] and Lienard [5] systems, and are an example of a dynamic system of fast-slow type [6], if its nonlinearity parameter is large.

In accordance with the magnetic nonlinearity and symmetry of the equation (5), the dynamo can be considered as a relaxation generator of the magnetic analogue of Higgs fields. The deflecting force in the equation (5) is cubic, and the potential will be a fourth-degree polynomial as in the Higgs equation. The nonlinear effect provides the magnetic field generation and the symmetry breaking. If the equation of magnetic induction does not have the magnetic symmetry of equation (5), then it cannot represent a dynamo. Primary dynamo models did not possess this symmetry.

Equations (5) are closed by the thermal diffusion equation

$$
\partial_{t} T+(\hat{G} \mathbf{f}, \nabla)\left(T+T_{0}\right)=k \Delta\left(T+T_{0}\right)
$$


where $k$ is the thermal conductivity coefficient, $T_{0}$ is the solution of this equation at $\mathbf{v}=0$, which corresponds to the stationary state of the system.

Thus, we obtained the magnetic dynamo equations (5) and (6) with magnetic feedback $\mathbf{f}$, which affects on the convection velocity through kinematic coefficient $\hat{G}$, which is mobility in (3) and feedback gain in (5) and (6).

In addition, the core of the integral operator $\hat{G}$ is convection waves, which are responsible for memory and nonlocality effects. Local approximation for $\hat{G}$ allows us to reduce the integral operator kinematic coefficients $\hat{D}$ and $\hat{G}$ to dynamo numbers that reduces the variables number and opens the way to small-dimention models with hidden kinematic dimensions. In the low-mode case with weak convection, this reducing leads to a simple dynamical system.

The equations (5) and (6) represent the magnetic-temperature dynemo waves. Kinematics is included implicitly in these equations through the operator kinematic coefficients $\hat{D}$ and $\hat{G}$ which are determened by complex friction (acoustic, Coriolis, turbulent, and viscous). Let us explain that acoustic or pressure waves carry away the impulse and act as effective friction.

In an incompressible medium, there are stationary waves of pressure that transfer momentum to the boundaries of the dynamo region with infinite speed.

We don't stop specially on boundary and other conditions for equations (1), (2), and (6), assuming that they provide a variety of solutions to these equations and actions of corresponding operators.

In order to obtain the magnetic dynamo equations (5) and (6), we used symbolic mathematics methods, which allow us to estimate the system parameters. We will do this for solar activity to show the effectiveness of the relaxation approach to the dynamo problem.

\section{Magnetic fields}

The condition $\mathbf{f}=0$ allows us to determine the configuration of the magnetic field, the Lorentz force of which is in balance with the Archimedes force. Depending on the speed of convection, nonlinear reversible oscillations of the magnetic field will be excited around this configuration.

In the case when the Archimedes and Lorentz forces are collinear and form a mutually orthogonal triple of vectors with $\mathbf{B}$ and $\operatorname{rot} \mathbf{B}=\mathbf{j}$, where $\mathbf{j}$ is the electric current, the condition $\mathbf{f}=0$ gives $B_{ \pm} \simeq \pm \sqrt{\rho \mu \mu_{0} g \beta T L / 2 \pi}$, where $L$ is the wave motion scale.

Taking into account $|T| \leq T_{0}$ (the saturation condition of the alternating variable $T$, which in amplitude cannot be greater than the stationary temperature $T_{0}$, otherwise the sum $T+T_{0}$ will take negative value) and the ratio $\beta \simeq 1 / T_{0}$ (as for ideal gas), we obtain the upper estimate for the Archimedes force $g \beta T \simeq g$ and the magnetic field $\hat{B}_{ \pm} \simeq \pm \sqrt{\rho \mu \mu_{0} g L / 2 \pi}$. Here $g L$ is the moment of gravitational forces per mass unit. It is responsible for magnetic field generation in a convective cell and it coincides in dimension with work per mass unit.

Substituting for the density of the solar atmosphere in the convection layer $\rho=10^{-3} \mathrm{~kg} / \mathrm{m}^{3}$ ( $10^{-3}$ from the Earth's atmosphere density), $\mu=1, \mu_{0}=4 \pi \cdot 10^{-7} \mathrm{H} / \mathrm{m}, \mathrm{g}=3 \cdot 10^{2} \mathrm{~m} / \mathrm{s}^{2}$ (acceleration of free fall on the Sun surface), and $L=D_{S}=1.4 \cdot 10^{9} \mathrm{~m}$ (the Sun diameter), we obtain $\hat{B}_{ \pm} \simeq \pm 10 T$.

This is four orders of magnitude greater than the observed fields on the sun's surface. If such intense fields are understood as internal, then the emergence of their flux tubes with high magnetic energy can explain sunspots, flares and coronal ejections on the Sun.

The condition $\mathbf{f}=0$ at $|B| \simeq \sqrt{\rho \mu \mu_{0} g L / 2 \pi}$ defines dynamical equilibrium for a free convection at the scale $L$. The kinematic approximation is applicable under the condition $|B| \ll \sqrt{\rho \mu \mu_{0} g L / 2 \pi}$ which gives the unlimitedly growing solution, so it can't be used to describe solar activity. 


\section{Oscillations}

On the period $T_{A}$ of solar activity, the magnetic field oscillates between the values $\hat{B}_{ \pm} \simeq$ $\pm \sqrt{\left(\rho \mu \mu_{0}\right) g L / 2 \pi}$. The oscillation factor $\lambda$ (cyclic gain and damping) is determined as $\lambda T_{A}=$ $2 \pi$. Amplification and damping $\lambda$, according to the induction equation (1), are determined by the distortion of the convection velocity $\lambda \simeq 2 \pi v / L$ (differential effect).

On the scale $L=D_{S}=1.4 \cdot 10^{9} \mathrm{~m}$, we have $\lambda \simeq 2 \pi v / D_{S}=2 \pi / T_{A}$. If $T_{A}=3.42 \cdot 10^{8} s(11$ years), then $v=D_{S} / T_{A} \simeq 4 \mathrm{~m} / \mathrm{s}$. It is the speed of meridian cerculation, $2 T_{A}$ is a full cycle with double pass $2 D_{S}$.

Taking into account the local, linear and isotropic approximation for (3) $\mathbf{v}=\hat{G} \mathbf{f} \rightarrow \mathbf{v} \simeq$ $G_{0} \mathbf{f}$ and the upper estimate $\left.\hat{f} \simeq f\right|_{B=0}=g \beta T \simeq g$ (see above $\beta T \simeq 1$ ), we find the mobility $G_{0} \simeq v / g \simeq 10^{-2} s$. This time (acceleration or free run) determines the damping and nonlinearity of the return force (or oscillation frequency) of the equation (5). The free run length at $v \simeq 4 \mathrm{~m} / \mathrm{s}$ will be about $10^{-2} \mathrm{~m}$.

Let us estimate the square of the oscillation frequency $\omega^{2}=|\operatorname{rot}(\hat{D} \hat{G}+\hat{1}) \mathbf{f}|$ in (5). If $\left.\hat{f} \simeq f\right|_{B=0}=g \beta T \simeq g, v \simeq G_{0} f$, and $\hat{D} \simeq 2 \Omega$ (predominance of the Coriolis effect on large convection scales is assumed), where $2 \Omega=4 \pi / T_{R}\left(T_{R}\right.$ is the Sun rotation period which is one month or $2.6 \cdot 10^{6} s$ ), the estimate of the first term in $\omega^{2}$ is $\omega_{D G}^{2}=\operatorname{rot} \hat{D} \hat{G} f \simeq 4 \pi \Omega v / L \simeq$ $4 \pi \Omega G_{0} g / L$.

We should note, $\omega_{D G} \simeq \sqrt{4 \pi \Omega v / L}$ is the geometric mean of zonal $\Omega$ and meridian $4 \pi v / L$ rotation velocities which presents the reletive magnetic oscillation frequency through the weak inclination of the rotation $\omega_{D G} / \Omega \simeq \sqrt{4 \pi v / L \Omega}$.

For $L=D_{S}, \omega_{D G}^{2}=\left(2 \pi / T_{D G}\right)^{2} \simeq 4 \pi \Omega G_{0} g / D_{S}=2 \Omega G_{0} \omega_{g}^{2}$, were $\omega_{g}^{2}=2 \pi g / D_{S}$ is the estimation of the second term in $\omega^{2}$. We see that $\omega_{g}^{2}=2 \pi g / D_{S}$ is the gravitational constant normalized on the scale $D_{S}$ and the square of the pendulum oscillations frequency in gravitational field. This is the result of the upper estimation of the Archimedes force.

Damping can be presented in these units as $\lambda=2 \pi v / D_{S}=2 \pi G_{0} g / D_{S}=G_{0} \omega_{g}^{2}$.

For $D_{S}=1.4 \cdot 10^{9} \mathrm{~m}$ and $T_{R}=2.6 \cdot 10^{6} \mathrm{~s}, T_{g}=2 \pi / \omega_{g}=2 \pi 10^{3} \mathrm{~s}$ (about 2 hours) and $T_{D G} \simeq 2 \cdot 10^{7} s(2 / 3$ year, this is the period of solar activity carrier oscillations).

Thus, we have three types of oscillations with the periods: $T_{A}, T_{D G}$ and $T_{g}$. They are a slow dynamo wave, a fast dynamo wave, and an internal gravity wave. We should note, there is very little time $G_{0}$. This is mobility in Stokes' law (3). So the scaling of solar activity is more than $10^{10}$.

The ratio $T_{D G} / T_{A}=1 / 16.5$ is a pitch of winding, vice versa $T_{A} / T_{D G}=16.5$ is the relative filling oscillation frequency in the relaxation pulse. These oscillations are observed in the Wolf numbers, figure 1 and the magnetic field, figure 2. About 16.5 oscillations per solar cycle is the fundamental result of the relaxation theory in the light of the universal dynamo paradigm.

In the composition of numbers $\omega_{D G}^{2} \simeq 4 \pi \Omega G_{0} g / D_{S}=2 \Omega G_{0} \omega_{g}^{2}$, the small dimensionless parameter $2 \Omega G_{0}$ of the evolutionary operator $\hat{D} \hat{G}$ is a phasa of Sun rotation on $2 G_{0}$ period. For two scales of the dynamo, we have $\omega_{D G}^{2} / \omega_{g}^{2} \simeq 2 \Omega G_{0} \ll 1$ that justifies the diffusion approximation for kinematic waves.

The composition of parameters $\omega_{D G}^{2} \simeq 4 \pi \Omega v / D_{S}$ can be also understood as the product of convection speed $v=G_{0} g$ and differential rotation $\Omega / D_{S}$. The latter value can be understood as a parameter of the omega effect. However, it should be noted that the representation of the magnetic induction equation (1) differs significantly from what was considered in [? ]. There is no full analogy with the dynamic numbers of this work. Here, we selected two main dynamo numbers $G_{0}$ and $\Omega / D_{S}$, which determine damping and nonlinear oscillation frequencies in (5). 


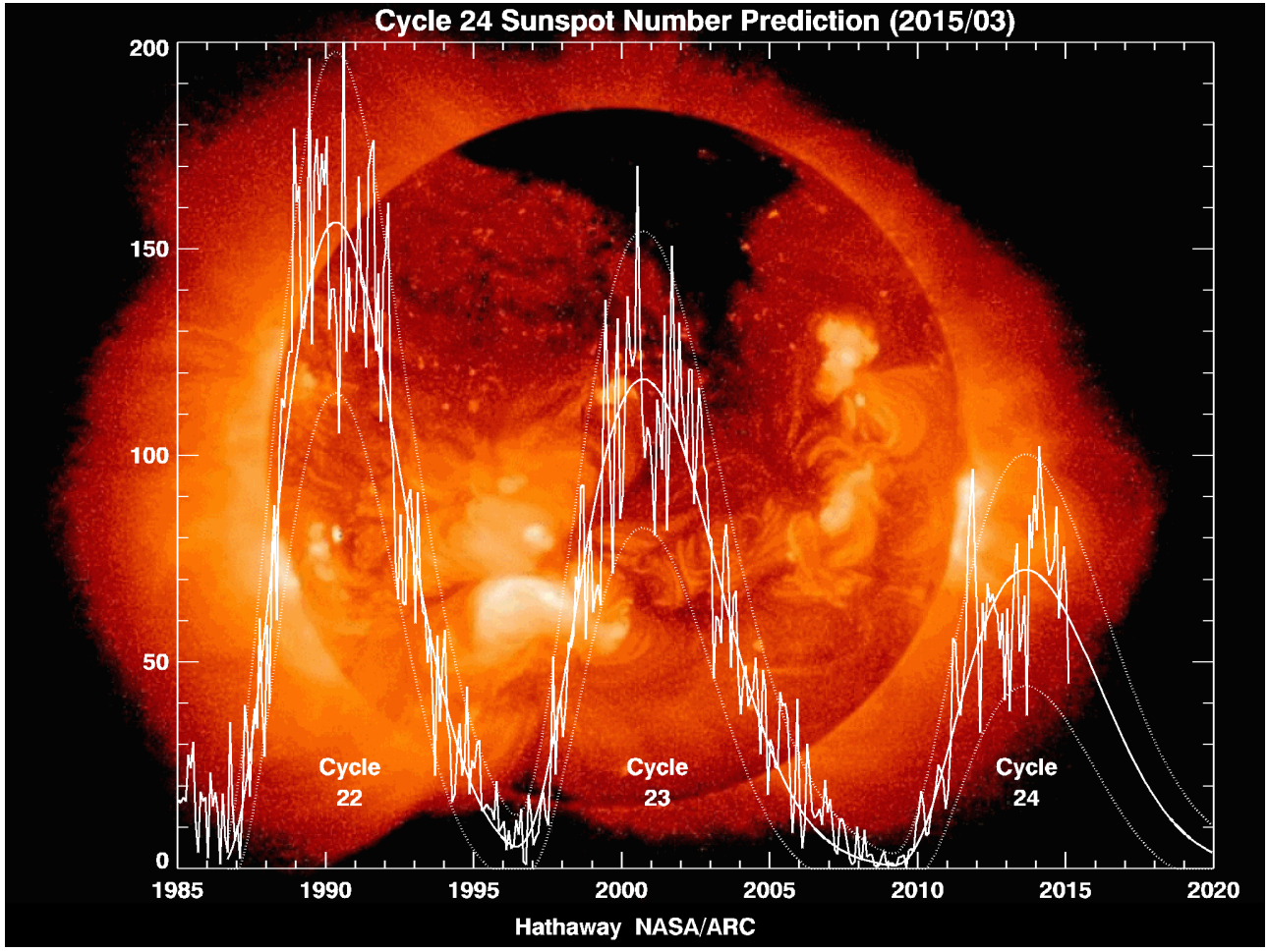

Figure 1. Oscillations in the solar cycle with a period of about $2 / 3$ years in the Wolf numbers.

\section{Nonlinearity parameter}

The nonlinearity parameter $\mu=\lambda / \omega_{D G}=T_{D G} / T_{A} \simeq \sqrt{\pi G_{0} g / \Omega D_{S}}$ is the winding pitch which must be more than a unit for the strong nonlinear mode. The relaxation impulse without filling is the sign of strong nonlinearity. This is a video impulse.

We should note that $\mu=\sqrt{2 \pi R o}$, were $R o$ is Rossby number.

For the Sun, $\mu \simeq 0.06$, therefore, the solar reversal oscillations are quasi-harmonic.

For more massive stars at the same diameter, the oscillations may be strong nonlinear.

If we turn to the scale $L<D_{S}$, for example to the sunspots scale $10^{-2} D_{S}$, where $\mu \simeq 0.6$, the oscillations there will be quasi-harmonic too.

But on the scale of $10^{3} \mathrm{~km}\left(10^{-3} D_{S}\right), \mu \simeq 2$. At a convection velocity of about $1 \mathrm{~m} / \mathrm{s}$, the Coriolis effects are comparable to kinematic turbulent ones on this scale.

Thus the non-linearity of oscillations increases when the scale decreases, and this is determined by kinematic turbulence which is the nonlinear component of complex friction in (3). As a result, the solar dynamo has a hybrid oscillation mode with inhomogeneous scaling, a quasi-harmonic oscillation mode at large scales, and a nonlinear mode at small scales. $10^{-3} D_{S}$ is the critical scale.

If we compare the magnetic dynamo with a violin then the magnetic field and the convective temperature wave are like the strings and a bow, and then a kinematic turbulence viscosity is like a rosin which affects the feedback gain. The kinematic variables play the role of the rosin. 


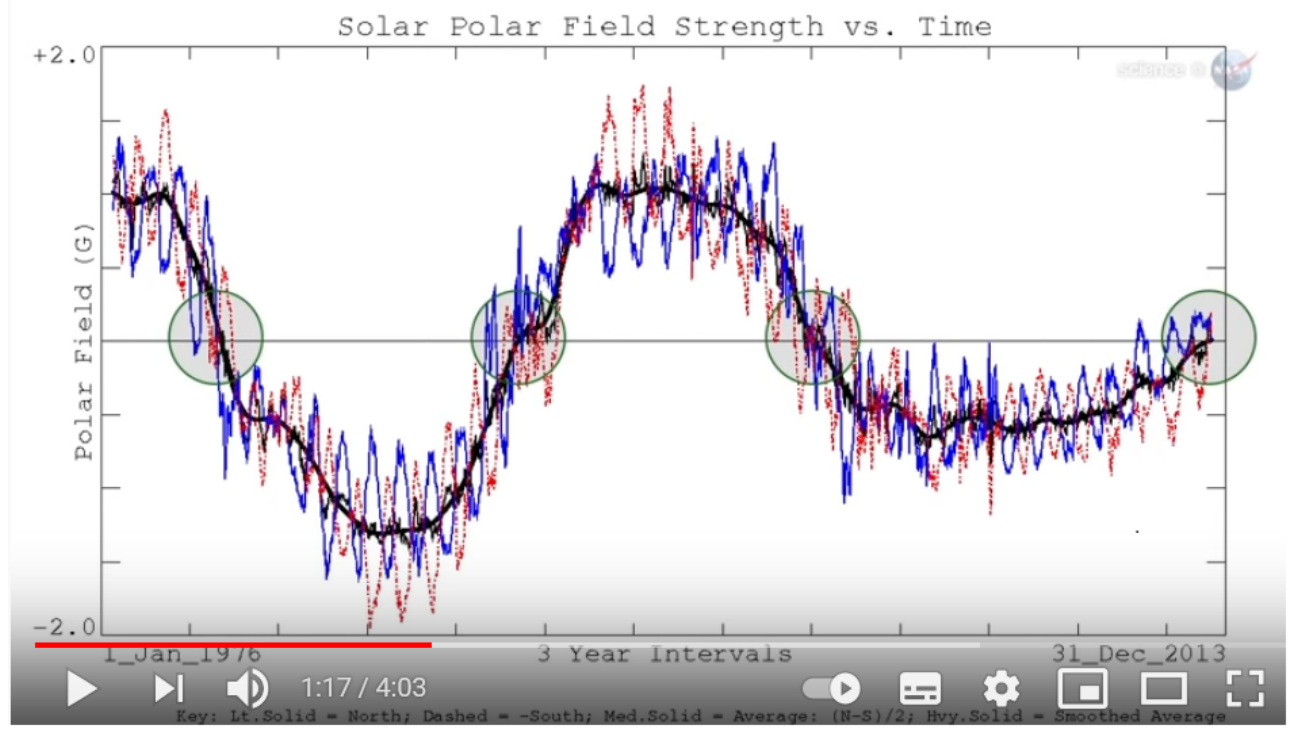

Figure 2. Oscillations in the solar cycle with a period of about 2/3 years in the magnetic field.

\section{Three regimes of convection and oscillations}

A nonlinear system will be of a fast-slow type if the nonlinearity parameter (the ratio of damping to oscillation frequency) is large. This is the case of the high convection speed $\mu^{2}=\pi G_{0} g / \Omega D_{S}=\pi v / \Omega D_{S}>1$ and strong nonlinear oscillations. Such a system represents fast changes which may be compared to the electric discharge.

The nonlinearity parameter $\mu^{2}=\pi v / \Omega D_{S}<1$ is small for weak convection. In this case, quasi-harmonic oscillations are realized. That can be compared with a low-mode laser. The slow amplitudes of such magnetic-temperature oscillations will obey the Lorentz-like dynamical system if we exclude fast changes by averaging over high frequency oscillations. Another way to obtian the equations for a low-mode dynamo is the Galerkin's method [2629]. But this method has a harmonic number selection problem that cannot be solved without a general relaxation approach.

Under very weak convection, the magnetic field is small and is determined by magnetic diffusion, and the reversible oscillations regress into excursus which are the oscillations about a stationary magnetic point without a reverse. Only in this case, the kinematic approximation for dynamo problem may be used. This is not applicable for solar activity but may be used for planetary dynamo [26-29].

The activation of very weak convection will determine the statistics of the waiting time of random reversals, between which there will be fading of reversible oscillations - this is the planetary dynamo mode. The minimum of Maunder solar activity - the fading of the reversible oscillations too.

Thus, three dynamo modes depending on convection are excursus, quasi-tonal and stron nonlinear ones. The two latter modes have reverses. The latter mode has fast reverse (magnetic switching).

Three dynamo modes may be compared with the same modes of brake pads, a violin, a spark gap, an earthquake, or a quantum generator. These comparisons help us to understand better how the dynamo works. 


\section{Reverse}

Two reversive dynamo modes differ by the sets of axial and nonaxial dipole components. For example, the axial reverse of the magnetic dipole is observed in solar activity [30], figure 3. While nonaxial magnetic dipole component is suppressed.

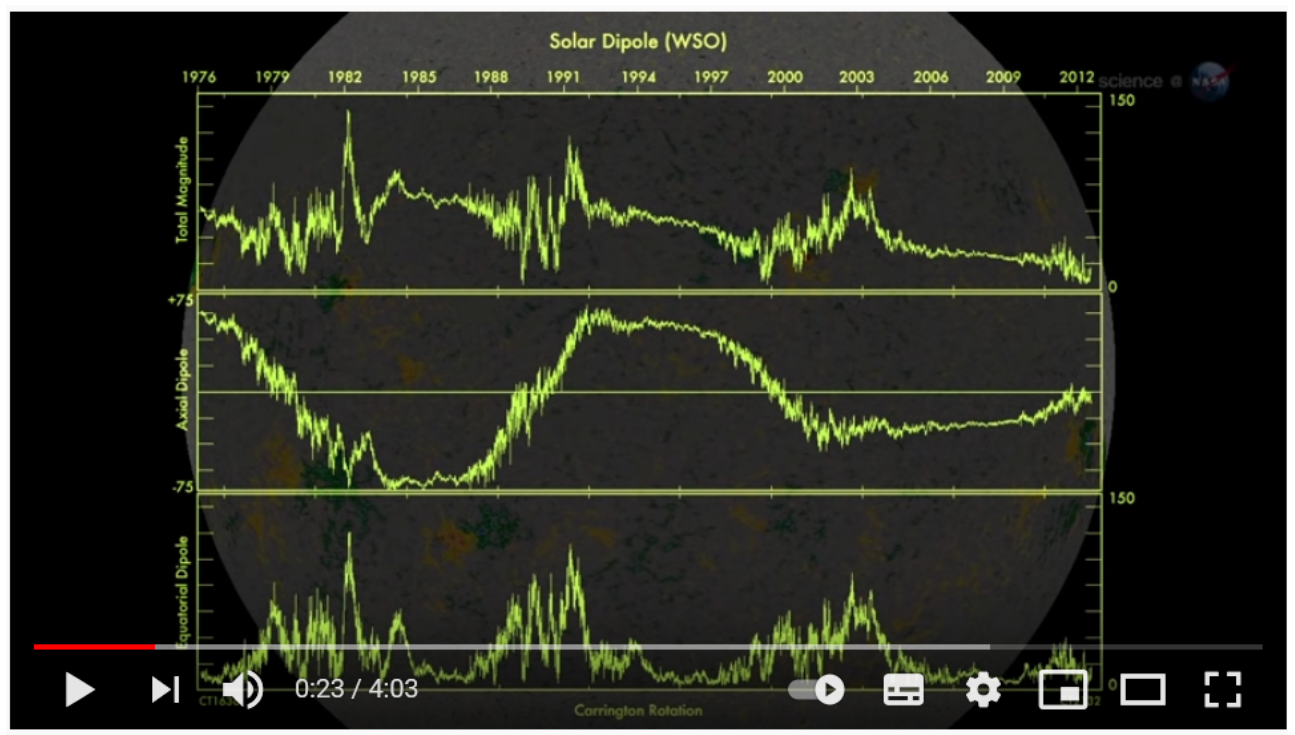

Figure 3. The ratio of the axial and non-axial dipole components of the magnetic field during reverse.

The synchronization of axial spheric harmonics in the defferent orders is responsible for the latitudinal localization of sunspots. It takes at least a hundred of spherical harmonics.

Assuming that only quasi axial modes are synchronized, Eq. (5) can be reduced to a onedimensional Lienard equation. However, this assumption is not correct for strong convection.

The magnetic dynamo in the strong convection may be compared with a quantum generator passive Q-switching mode or seismic unloading [31,32]. Such fast reverse is a magnetic switching or fall. This is the generating mechanism of a superstar, quasar or Black Hole.

All relaxation oscillations have a specific shape of Berlage impulse which is also in the solar activity, see figure 1 .

\section{Conclusion}

The new dynamo paradigm is a special representation of the dynamo by a magnetic analogue of Higgs fields.

The magnetic dynamo is presented by the Lienard relaxation oscillator with the cubic nonlinearity of restoring force corresponding to the potential of the Mexican hat or champagne bottle which is used to determine the Higgs fields.

Strongly nonlinear oscillations in the magnetic analogue of Higgs fields represent catastrophe events.

The dynamo effect can be used in field theory as an alternative to spontaneous symmetry breaking.

The relaxation dynamo mechanism is the balance between Archimedes and Lorentz forces. This is the key to understanding the dynamo paradigm. 
The dynamo has three oscillation modes depending on convection speed, they are excursus, quasi-harmonic and strong nonlinear ones.

The dynamo numbers are estimated for the solar activity and it was shown that Sun magnetic oscillations are quasi-harmonic for large scales and critical for small scales.

Oscillations in the solar cycle with a period of about $2 / 3$ years are a fundamental result of the new dynamo paradigm.

The relaxation approach to the dynamo problem gives the explanations of numerous small-dimension and low-mode models, the variety of which is generated by the properties of kinematic coefficients and their approximations which should be reviewed from a general point of view.

Sudden changes in the properties of the kinematic coefficients can give stable structures or cause local disasters. It's like a loss of strength in a solid. This is the key to understanding the catastrophes of living, weather and climatic systems.

The relaxation approach provides a comprehensive understanding of the magnetic dynamo and it may be useful for many areas of Physics, especially where the fast-slow nonlinear dynamical systems are considered to describe catastrophic events.

The representation of the dynamo by magnetic-temperature waves reduces the initial dimension and gives the effective approximation method in the study of nonlinear systems.

The dynamo paradigm can be generalized to any fields.

Then the dynamo effect can explain the scaling and baryon asymmetry of the Universe.

The elementary particles can be considered as the space and time dislocations on the internal scale, as the catastrophes of the fold type.

A better understanding of subhadronic structures can be achieved through the examples of solar activity and many other relaxation systems.

The collapse and inflation of dark and light matter at different scales of the Universe can be presented as turbulent pulsations with disasters.

The Universe in the light of the catastrophes theory can become an alternative to the concept of the Big Bang.

\section{Acknowledgments}

The paper was carried out on the subject Physical Processes in the System of Near Space and Geospheres under Solar and Lithospheric Impacts: AAAA-A 21-121011290003-0.

\section{References}

[1] B. M. Shevtsov, E3S Web of Conferences 196, 02026 (2020)

[2] B. van der Pol, The London, Edinburgh, and Dublin Philosophical Magazine and Journal of Science Ser.7, 2978 (1926)

[3] B. van der Pol, The London, Edinburgh, and Dublin Philosophical Magazine and Journal of Science Ser.7, 365 (1927)

[4] B. van der Pol, J. van der Mark, Nature 120363 (1927)

[5] A. Liénard, Etude des oscillations entretenues. Revue générale de l'électricité 23901 and 946 (1928)

[6] M. Diener, The Mathematical Intelligencer 638 (1984)

[7] E. C. Bullard, H. Gellman, Philosophical Transactions of the Royal Society of London A 247213 (1954)

[8] E. C. Bullard, Proc. Cambridge Philos. Soc. 51744 (1955)

[9] T. Rikitake, Proceedings of the Cambridge Philosophical Society 5489 (1958) 
[10] E. N. Parker, Astrophysical Journal 122293 (1955)

[11] H. W. Babcock, Astrophysical Journal 133 572Â (1961)

[12] Max Steenbeck, Fritz Krause, Karl-Heinz Rädler, Z. Naturforsch 21a 369 (1966)

[13] R. B. Leighton, Astrophysical Journal 1561 (1969)

[14] N. I. Kliorin, A. A. Ruzmaikin, Magn. hydrodyn. 18116 (1982)

[15] K. M. Kuzanyan, D. D. Sokoloff, Astron. Rep. 40249 (1996)

[16] N. Meunier, M. R. E. Proctor, D. D. Sokoloff, A. M. Soward, S. M. Tobias, Geophys. Astrophys. Fluid Dyn. 86249 (1997)

[17] A. P. Bassom, K. M. Kuzanyan, A. M. Soward, Proc. R. Soc. Lond. A 4551443 (1999)

[18] Ya. B. Zel'dovich, A. A. Ruzmaikin, Sov. Phys. JETP 51493 (1980)

[19] A. A. Ruzmaikin, Comments on Astrophysics 985 (1981)

[20] Ya. B. Zeldovich, A. A. Ruzmaikin, Astrophys. and Space Phys. Rev. 2333 (1983)

[21] N. O. Weiss, F. Cattaneo, C. A. Jones, Geophys. Astrophys. Fluid Dyn. 30305 (1984)

[22] C. A. Jones, N. O. Weiss, F. Cattaneo, Physica. Ser. D 14161 (1985)

[23] Ya. B. Zeldovich, A. A. Ruzmaikin, UFN 152263 (1987)

[24] P. Charbonneau, Annual Review of Astronomy and Astrophysics 52251 (2014)

[25] H. P. Popova, UFN 186577 (2016)

[26] G. M.Vodinchar, B. M. Shevtsov, Vychisl. Tekhnol. 143 (2009)

[27] G.M. Vodinchar, L.K. Kruteva, Computational Technologies 1635 (2011)

[28] L. K. Feschenko, G. M. Vodinchar, Nonlin. Processes Geophys. 22361 (2015)

[29] L. K. Feschenko, G. M. Vodinchar, Magnetohydrodynemics 52287 (2016)

[30] NASA, The Sun's Magnetic Field is About to Flip (2013) http://science.nasa.gov/science-news/...

[31] R. Burridge, L. Knopoff, Bull. Seismol. Soc. Am. 57341 (1967)

[32] J. M. Carlson, J. S. Langer, Phys. Rev. Lett. 622632 (1989) 